\title{
Gewichtszunahme bei Kindern während Corona-Lockdown
}

\author{
Aktuelle Erhebungsdaten des wissenschaftlichen Präventi- \\ onsprojektes EDDY belegen deutliche Zunahme an Fettmasse \\ bei Kindern während Corona-Lockdown.
}

EDDY, derzeit das einzige evidenz-basierte, wissenschaftlich begleitete Projekt in Österreich zur Prävention von Übergewicht bei Kindern, war aufgrund des Corona-Lockdowns gezwungen, die vorgesehenen schulischen Interventionen in der untersuchten Interventionsgruppe auszusetzen. Das Projektteam rund um den Ernährungsmediziner Univ.-Prof. Dr. Kurt Widhalm nutzte die Gelegenheit und dokumentierte im Projektzeitraum auch diese Pause. Es stehen damit erstmals valide Gewichtsdaten zum Corona-Lockdown zur Verfügung. Die Ergebnisse sind bemerkenswert.

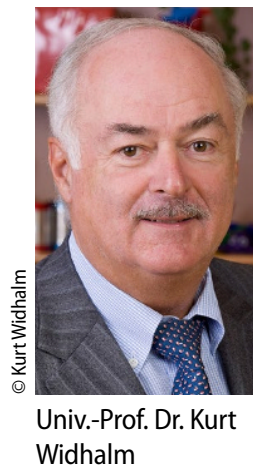

Quelle: Presseaussendung der ÖIAE, Mag. Johannes Martschin, 08.07.2020

\section{Änderungen wegen Pandemie}

Die für ein halbes Jahr geplante Intervention konnte aufgrund der Corona Maßnahmen nur sieben Wochen durchgeführt werden. Danach pausierten die Interventionen in physischer Präsenz, doch erhielt die Interventionsgruppe während des Home Schoolings Material für Ernährungsund Sportinterventionen. Die Kontrollgruppe erhielt keine Informationen.

Die Messungen von Körpergewicht und Körperzusammensetzung wurden am Schulschluss durchgeführt. Sie zeigen - im Unterschied zu früheren Untersuchungen, bei denen eine permanente Intervention bei Vorhandensein einer Kontrollgruppe ohne Intervention stattfand, - , dass die Kinder in der Corona-Phase ohne Intervention deutlich mehr an Fettmasse zuge-

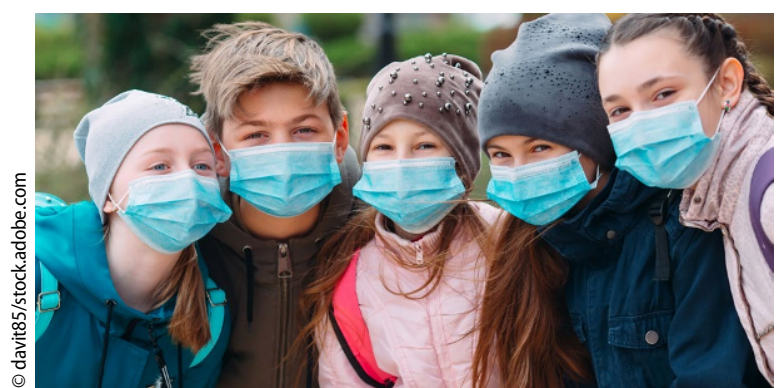

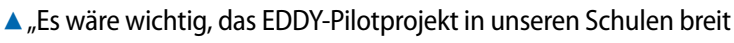
umzusetzen", so Widhalm (Symbolbild mit Fotomodellen)

Das Österreichische Akademische Institut für Ernährungsmedizin (ÖAIE) wurde 1996 auf Initiative des damaligen Präsidenten der Ärztekammer, Prim. Dr. Michael Neumann, mit dem Ziel gegründet, Ärztinnen und Ärzte im Fach der Ernährungsmedizin fortzubilden. Das ÖAIE ist interdisziplinär ausgerichtet und vereint unter der Leitung von Univ.-Prof. Dr. Kurt Widhalm Expertinnen und Experten aus den Bereichen der Medizin, Psychologie, Ernährungswissenschaften, Diätologie, Sportwissenschaften und Nahrungsmittelproduktion. Als führende Fortbildungs- und Forschungs-Institution für Ernährungsmedizin in Österreich richtet es regelmäßig wissenschaftliche Veranstaltung aus und publiziert vierteljährlich das „Journal für Ernährungsmedizin“. Das EDDY-Programm ist derzeit das einzige evidenz-basierte, wissenschaftlich begleitete Projekt in Österreich zur Prävention von Übergewicht bei Kindern. Das vom ÖAIE initiierte Projekt wird in einer Volksschule im 12. Wiener Gemeindebezirk seit dem Wintersemester 2016/17 durchgeführt.

nommen hatten als in der Vorperiode. Die Zahl an Adipösen stieg in beiden Gruppen deutlich an. Die online durchgeführten Informationen in der Interventionsgruppe zeigten jedoch Wirkung durch eine geringere Zunahme an Übergewicht als in der Kontrollgruppe.

„Die Ergebnisse zeigen, dass das Ausbleiben einer professionell durchgeführten Ernährungs- und Sportintervention - aufgrund der CoronaMaßnahmen - zu einem sprunghaften Anstieg der Zahl an adipösen Kindern geführt hat", erläutert Studienleiter Kurt Widhalm. „Sie zeigen aber auch, wie wichtig es wäre, das EDDY-Pilotprojekt in unseren Schulen breit umzusetzen", sagt Widhalm. „Denn derzeit werden die klaren WHO-Vorgaben zur Prävention von Übergewicht in Öster- reich schlicht und ergreifend einfach nicht umgesetzt", so Widhalm.

Das dreijährige EDDY-Projekt wurde mit Sommersemester 2020 abgeschlossen. Die Ergebnisse werden voraussichtlich im Herbst 2020 präsentiert.

\section{Weitere Informationen:}

https://www.oeaie.org/

Hinweis des Verlags. Der Verlag bleibt in Hinblick auf geografische Zuordnungen und Gebietsbezeichnungen in veröffentlichten Karten und Institutsadressen neutral.

Paediatr. Paedolog. 2020 · 55:268 https://doi.org/10.1007/s00608-02000836-8

(c) Springer-Verlag GmbH Austria, ein Teil von Springer Nature 2020 